\title{
Моделирование процесса усадки термоэлектриков при искровом плазменном спекании на примере $\mathrm{Ge}-\mathrm{Si}$
}

\author{
(C) А.С. Тукмакова, К.Л. Самусевич, А.В. Новотельнова, И.Л. Тхоржевский, Е.С. Макарова
}

Университет ИТМО,

197101 Санкт-Петербург, Россия

E-mail: astukmakova@corp.ifmo.ru

Поступила в Редакцию 7 февраля 2019 г.

В окончательной редакции 10 фревраля 2019 г.

Принята к публикации 14 фревраля 2019 г.

\begin{abstract}
Проведено моделирование усадки термоэлектрического материала на основе $\mathrm{Ge}-\mathrm{Si} p$-типа в процессе искрового плазменного спекания. Моделирование выполнено методом конечных элементов в программе Comsol Multiphysics. Модель механических процессов была основана на результатах ранее опубликованных работ, посвященных моделированию спекания порошковых металлических материалов и керамик. Описание механических процессов включало упругую и пластическую деформацию обрабатываемого материала. В процессе расчета учитывалось влияние пористости материала на тепловые, электрические и механические свойства. Проведен расчет давления спекания в образце. Рассчитано изменение диаметра образца при выдержке при максимальной температуре.
\end{abstract}

DOI: $10.21883 /$ FTP.2019.06.47728.37

\section{1. Введение}

Одним из наиболее распространенных методов обработки порошковых материалов является искровое плазменное спекания (ИПС) [1]. ИПС позволяет компактировать порошки при помощи пропускания импульсного тока и приложения механического давления.

Структуру и свойства материала, получаемого в процессе ИПС, определяют температура и давление спекания. Экспериментальное измерение этих параметров непосредственно в образце затруднительно.

Моделирование ИПС позволяет проводить расчет полей температуры и давления. Однако, несмотря на рост числа экспериментальных и численных исследований, модели, полностью описывающей все микро- и макропроцессы ИПС, до сих пор не создано.

Удобным и современным методом компьютерного моделирования является метод конечных элементов (МКЭ). В макромодели установки ИПС возможно использование дополнительного модуля, позволяющего аналитически учитывать изменение пористости материала и размера зерна. Расчеты с использованием МКЭ в совокупности с дополнительными уравнениями для микропроцессов представлены на примере спекания порошков металлов и керамик [2-6].

Существуют работы по моделированию распределения температуры и давления, расчета эффекта Пельтье при спекании термоэлектриков [7,8]. Изменение пористости, плотности и размеров образца оказывает значительное влияние на тепловые, электрические и механические процессы ИПС. Однако отсутствуют работы, представляющие анализ усадки и деформации термоэлектрических материалов и учитывающие влияние пористости на свойства образца.
В данной работе представлены результаты расчета давления спекания термоэлектрического материала на примере сплавов $\mathrm{Ge}-\mathrm{Si}$ р-типа с использованием математической модели механических процессов, предложенной в работе [4] и позволяющей учитывать переход от упругой к пластической деформации. Модель являлась нестационарной 2D осесимметричной. Описание установки ИПС и свойств используемых материалов, а также система электрических и тепловых уравнений аналогичны ранее описанным нами в работе [8]. В основе модели лежит система дифференциальных уравнений, включающая описание электрических, тепловых и механических процессов. Модель учитывает термоэлектрический эффект, джоулев нагрев и тепловое расширение.

Термоэлектрические свойства $\mathrm{Ge}-\mathrm{Si}$ и параметры эксперимента (давление, ток) были взяты из работы [9] в виде температурных зависимостей. Коэффициенты тепло- и электропроводности рассматривались в качестве функций относительной плотности. Модель, учитывающая влияние пористости на тепло- и электропроводность, представлена в работах $[10,11]$.

Для учета влияния пористости на механические свойства коэффициент Пуассона и модуль Юнга также описывались в виде функций относительной плотности [12-14]. Были приняты во внимания результаты работы [14], в которой было показано, что пористость имеет одинаковое влияние на относительную величину модуля Юнга (максимальная величина модуля Юнга соответствовала материалу с теоретической плотностью) для металлов и керамик. Таким образом, было допущено, что зависимость модуль Юнга и коэффициент Пуассона от пористости для термоэлектрического материала будет совпадать с представленной в работе [14].

Механические свойства сплава $\mathrm{Ge}-\mathrm{Si}$ были взяты из работы [15]. Зависимость коэффициента теплового 
расширения от температуры была взята из работы [16]. Зависимость относительной плотности образца от времени спекания представлена на рис. 1. Теоретическая плотность $\mathrm{Ge}-\mathrm{Si}$ была принята равной 2860 кг/м³. На рис. 1 представлена экспериментальная зависимость усадки образца от времени. Начальная высота образца составляла 2.58 мм, конечная составила 2 мм. Смещение верхней границы образца было ограничено в вертикальном направлении. При этом верхняя граница могла смещаться в горизонтальном направлении. Все остальные поверхности могли смещаться в любом направлении под действием теплового расширения и давления со стороны других элементов.

\section{2. Результаты}

Был проведен расчет нагрева образца (см. рис. 1) за счет выделения теплоты Джоуля при прохождении тока через элементы установки и образец. На конечную величину температуры спекания оказывали влияние эффект Пельтье, процессы теплообмена между образцом, элементами установки и вакуумной камерой.

На рис. 2 представлено распределение давления по высоте образца при разных значениях радиуса. Почти для всех кривых, кроме кривой с координатой 6.3 мм, характерен рост давления с увеличением высоты образца, т.е. при приближении к верхней поверхности, ограниченной в смещении по вертикальной оси. Для точек, находящихся в одной горизонтальной плоскости, наблюдается разница в величине расчетного давления. Наибольшая разница давления в верхней плоскости образца составила $3.5 \mathrm{MПа,} \mathrm{в} \mathrm{нижней} \mathrm{-} 2.7$ МПа. Максимальная разница давления по высоте образца достигает 4 МПа.

На рис. 3 представлена радиальная составляющая поля смещения в образце. Во время спекания происходит

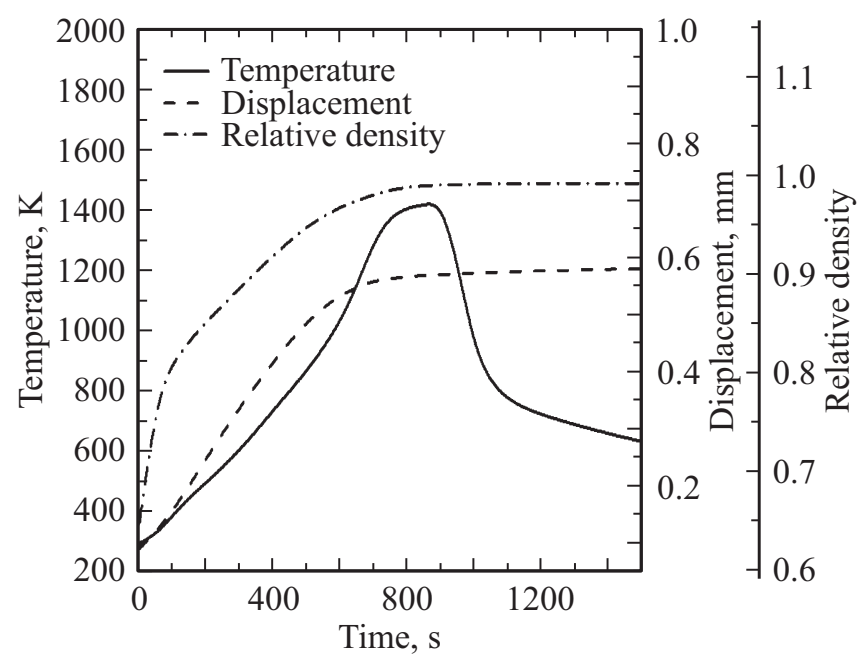

Рис. 1. Зависимость усадки образца, относительной плотности и средней температуры спекания образца от времени спекания. Величина температуры получена расчетным путем.

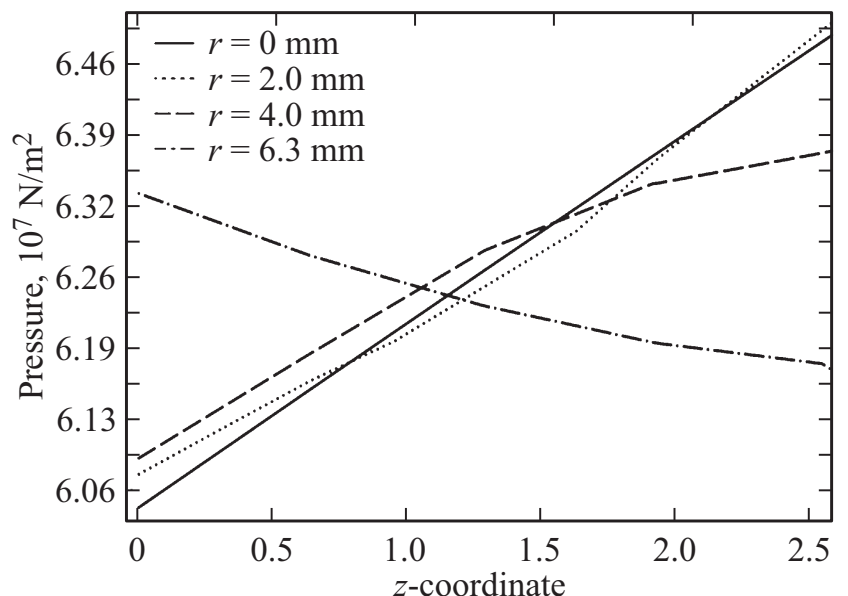

Рис. 2. Давление в образце в зависимости от высоты и радиальной координаты в момент времени $900 \mathrm{c}$.

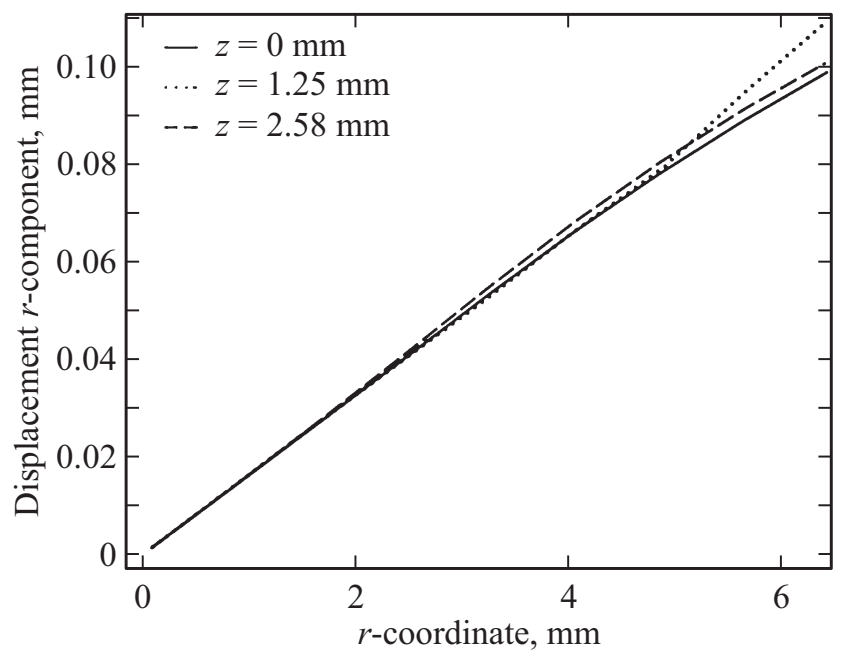

Рис. 3. Радиальная составляющая поля смещения в образце.

сжатие образца за счет приложения внешней силы в совокупности с расширением элементов установки и самого образца при нагреве. В соответствии с расчетом, представленным в данной работе, такое комплексное механическое воздействие на обрабатываемый образец приводит к его расширению в радиальном направлении на величину, достигающую 0.11 мм. Результаты, показанные на рис. 3, представлены для половины образца, следовательно, суммарное увеличение диаметра образца составляет $0.22 \mathrm{Mм}$, что соответствует $1.7 \%$ от исходного диаметра образца на начальной стадии спекания.

\section{3. Заключение}

Проведено моделирование процесса усадки термоэлектрического материала с последующим расчетом давления спекания и изменения геометрических размеров образца в момент выдержки. 
Модель апробирована на примере спекания порошков сплава $\mathrm{Ge}-\mathrm{Si}$. Расчетное давление неоднородно в объеме образца. Величина давления изменяется в пределах от 60.1 до 65.2 МПа. В среднем расчетная величина давления выше, чем экспериментальная, равная $60 \mathrm{MПа} \mathrm{[9].} \mathrm{Возможной} \mathrm{причиной} \mathrm{получения} \mathrm{более}$ высоких значений давления может быть использование при моделировании идеализированных контактов между элементами установки и образцом.

Проведен расчет изменения размеров образца в радиальном направлении при спекании. Показано увеличения диаметра образца на $1.7 \%$ за счет теплового расширения и осевого сжатия.

Исследование выполнено при финансовой поддержке РФФИ в рамках научного проекта № 18-38-00371.

\section{Список литературы}

[1] O. Guillon, J. Gonzalez-Julian, B. Dargatz, B. Dargatz, T. Kessel, G. Schierning, J. Räthel, M. Herrmann. Adv. Eng. Mater., 16, 830 (2014).

[2] W. Wang, H. Qi, P. Liu, Y. Zhao, H. Chang. Metals, 8 (7), 537 (2018).

[3] P. Han, X.Z. An, Y.X. Zhang, Z.S. Zou. J. Min. Metall. Sect. B-Metall., 51, 163 (2015).

[4] Y. Song, Y. Li, Z. Zhou, Y. Lai, Y. Ye. J. Mater. Sci., 46, 5645 (2011).

[5] C. Manière, L. Durand, A. Weibel, C. Estournès. Acta Mater., 102, 169 (2016).

[6] P. Mondalek, L. Silva, M. Bellet. Adv. Eng. Mater., 13 (7), 587 (2011).

[7] Giovanni Maizza. J. Mater. Sci., 52, 10341 (2017).

[8] L.P. Bulat, A.V. Novotelnova, A.S. Tukmakova, D. Yerezhep, V.B. Osvenskii, A.I. Sorokin, V.P. Panchenko, L.V. Bochkov, S. Asmontas. J. Electron. Mater., 47 (2), 1589 (2018).

[9] A. Usenko, D. Moskovskikh, M. Gorshenkov, A. Voronin, A. Stepashkin, S. Kaloshkin, D. Arkhipov, V. Khovaylo. Scripta Mater., 127, 63 (2017).

[10] D. Wang, Y. Wu, M. Jiao, J. Yu, T. Xie, Y. Yin. Powder Metall Technol., 26, 88 (2008).

[11] J.M. Montes, F.G. Cuevas, J. Cintas. Metall. Mater. Trans. B Proc. Metall. Mater. Proc. Sci., 38, 957 (2007).

[12] Y. Li, P. Chen, W. Xia, Z. Zhou, W. Li. Trans. Nonferrous. Metal. Soc., 16 (3), 507 (2006).

[13] Y. Song, Y.Y. Li, Z.Y. Zhou, Z.X. Zheng, P.Q. Chen. Trans. Nonferrous. Metal. Soc., 20, 1470 (2010).

[14] A. Fedotov. J. Mater. Sci., 52, 2964 (2017).

[15] J.R. Gladden, G. Li, R. Adebisi, S. Firdosy, T. Caillat, V. Ravi. Phys. Rev. B, 82, 045209 (2010).

[16] Glen A. Slack, S.F. Bartram. J. Appl. Phys., 46, 89 (1975).

Редактор А.Н. Смирнов

\section{Simulation of thermoelectric materials densification during spark plasma sintering with the example of $\mathbf{G e}-\mathbf{S i}$}

\section{A.S. Tukmakova, K.L. Samusevich, A.V. Novotelnova, I.L. Tkhorzhevskiy, E.S. Makarova}

ITMO University, 197101 St. Petersburg, Russia

Abstract The densification of $\mathrm{Ge}-\mathrm{Si}$ within spark plasma sintering has been simulated. The simulation was based on finite elements method and implemented in Comsol Multiphysics. The model of mechanical processes was based on the simulation results published earlier for metal and ceramic powder materials. The model included plastic and elastic deformation description, impact of porosity on electrical, thermal and mechanical properties. The pressure field in the sample has been calculated. The change of sample diameter due to the expansion and compression has been obtained for the soaking period. 